- Demonstrates the need for communication and teamwork not only between orthodontist and patient but between orthodontist, patient and general dental practitioner.

- Demonstrates that teamwork is not only required pre-treatment but also during treatment and possibly long-term.

- Demonstrates the need for the general dental practitioner to be aware of the risks of orthodontic treatment in order to discuss and manage patients better prior to and after orthodontic referral.

\title{
Teamwork in orthodontics: Limiting the risks of root resorption
}

\author{
F. Luther, ${ }^{1}$ S. Dominguez-Gonzalez ${ }^{2}$ and S. A. Fayle ${ }^{3}$
}

Orthodontic treatment is not without risk. The risks may be due to patient factors (which may not always be evident before treatment) or may come about because of the treatment itself. While the common types of risk are well documented, less information is available as to how some of the more unusual problems can best be managed when they arise; often the need for teamwork between the patient, orthodontist and general dental practitioner (GDP) are underestimated. This paper presents three patients in whom various root-related problems existed either before orthodontic treatment or which arose during orthodontic treatment; demonstrates how they were managed; and highlights the need for teamwork to ensure a 'least harmful' outcome. All patients were followed up for over a year.

\section{INTRODUCTION}

As with any dental procedure, orthodontic treatment is not exempt from risks or complications. A well-recognised complication of orthodontic treatment is root resorption, and while the established literature is replete with various factors that may be related to root resorption, ${ }^{1-7}$ definitive predictors are still lacking. However, as well as the risks that apply to every course of orthodontic treatment, there may also be limits imposed by the patient, for example pathological conditions involving dental trauma or lack of cooperation. This article presents three patients who underwent orthodontic treatment and who demonstrated before, during or after the treatment different complications in one or more upper incisors, all of them being root resorption related. The limits and risks associated with their orthodontic treatment are discussed and the need for teamwork between the patient, orthodontist and the patient's GDP are highlighted.

${ }^{1 *}$ Senior Lecturer and Honorary Consultant in Orthodontics, Department of Orthodontics, ${ }^{2} \mathrm{FTTA}$ in Orthodontics, ${ }^{3}$ Consultant in Paediatric Dentistry, Leeds Dental Institute.

"Correspondence to: Dr Friedy Luther, Leeds Dental Institute, University of Leeds, Clarendon Way, Leeds, LS2 9LU Email to:ja.gm@btinternet.com

\section{Refereed Paper}

Received 30.01.03; Accepted 05.07.04

doi: 10.1038/sj.bdj.4812188

() British Dental Journal 2005; 198: 407-411

\section{CASE REPORTS}

\section{Case 1}

A 10-year-old girl presented with a skeletally based class II division 1 malocclusion. Cephalometrically, she had a skeletal II pattern associated with average vertical dimensions. The lower lip was positioned behind the upper incisors. Intra-orally, the upper and lower incisors were proclined, the overjet was $8.5 \mathrm{~mm}$ and the overbite was increased and incomplete (Fig. 1). All teeth were erupted except for the third molars and both arches were well aligned with the exception of the upper incisors. She admitted being a digit sucker and there was also a history of previous dental trauma, the upper central incisors having been damaged three years earlier. Previous records indicated that UL1 (21) had been intruded and suffered an apical third root fracture with displacement of the coronal fragments. UR1 (11) was extruded and the root length was reduced (Figs 2a, 2b). Sensibility tests indicated that the coronal fragment of UL1 (21) was vital. UR1 (11) also gave a positive response to sensibility tests. Radiographically, this tooth showed significant pulpal obliteration which was consistent with the continued vitality of the pulp.

A paedodontic opinion suggested that the prognosis of both incisors was good, although orthodontic movement was deemed to probably carry a higher than normal risk of inducing root resorption. It was therefore advised that the upper central incisors be radiographically mon-

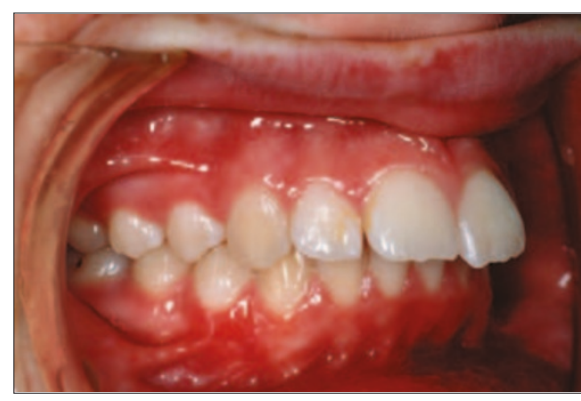

Fig 1 Case report 1: pre-treatment photograph.

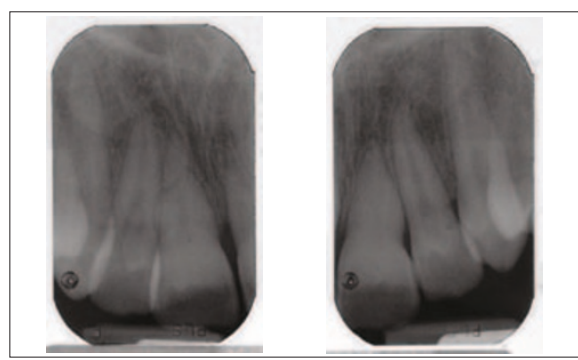

Fig 2a, 2b Case report 1 : pre-treatment periapical radiographs.

itored every three to four months during orthodontic treatment; the vertical discrepancies between the upper incisor edges were also to be monitored in case changes occurred suggestive of ankylosis. The treatment plan was to use a TwinBlock functional appliance (incorporating a palatal screw and z-springs to procline UL2 (22) and UR2 (12)) to correct the overjet, followed by fixed appliances in both arches. 


\section{PRACTICE}

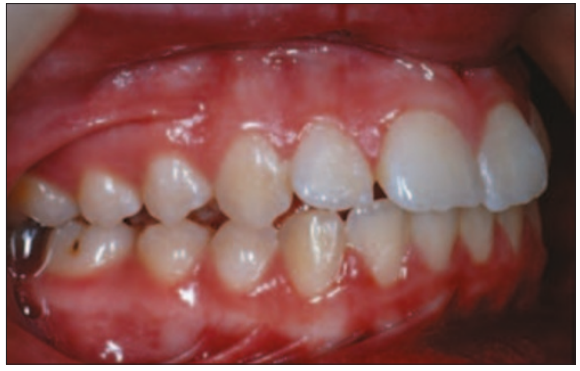

Fig 3 Case report 1 : post-treatment photograph.

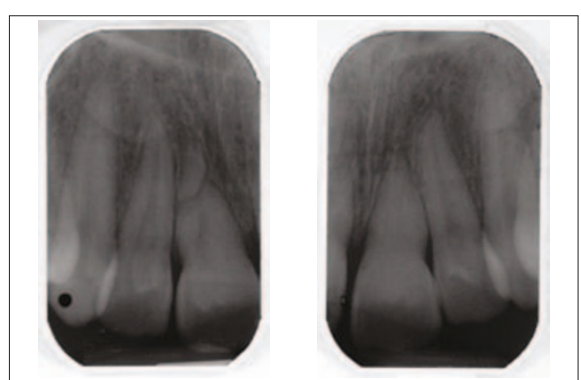

Fig 4a, 4b Case report 1: 6 months posttreatment periapical radiographs.

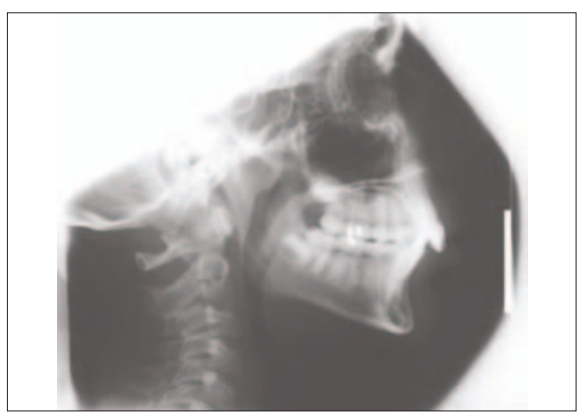

Fig 5 Case report 2: pre-treatment lateral cephalogram.

The patient wore the Twin-Block appliance full-time. During treatment, periapical radiographs were taken as described combined with vitality tests to assess the progress of UL1 (21) and UR1 (11): no significant changes were seen. After nine months, an overjet of $1 \mathrm{~mm}$ and overcorrection of the molars was achieved. The patient was asked to wear the now passive appliance at night only for another two months. Then, the Twin-Blocks were replaced by an upper Hawley Retainer at night for six months and alternate nights for another six months. After 23 months the treatment was completed. The molars were Class I and the overjet was $3 \mathrm{~mm}$ (Fig. 3). The vertical discrepancies between the upper incisors edges did not change during the treatment. The repeat radiographs showed that the apical and coronal fragments of UL1 (21) had separated with 'rounding' resorption at the line of the fracture, as was expected (Figs 4a, 4b).

The patient was pleased with the results and declined final alignment with fixed appliances. Subsequent follow-up showed her occlusion to be stable. Vitality tests were positive for all upper incisors and there were no signs of tooth mobility. UL1 (21) and UR1 (11) presented pulpal obliteration but no other pathologies were associated. The patient was discharged from the department to her GDP with advice regarding long-term monitoring of the upper incisors with periapical radiographs every five years.

The prognosis of a root fractured tooth during orthodontic treatment depends on the type of healing response and the location of the fracture. ${ }^{8}$ Root fractured permanent teeth may heal by one of three modalities: by hard tissue union, by interposition of connective tissue or by interposition of bone and connective tissue. In the latter two situations, the coronal fragment is no longer connected to the apical fragment and then, as in Case 1, where the root fragments are separated by connective tissue the tooth can be moved orthodontically but is treated as a tooth with a shortened root. Furthermore, in this patient the fracture had occurred in the apical third. This tends to improve the prognosis due to the improved periodontal support at this level.

However, during this orthodontic tooth movement, as well as the root fragments undergoing separation, rounding of the fracture edges and slight shortening, the pulp (as occurred here) may also undergo obliteration - a finding which has been reported previously. ${ }^{8}$ Nevertheless, other problems can also arise during orthodontic treatment. For example, ankylosis, loss of vitality of the coronal fragment, periapical pathology and/or greater root resorption. Treatment options for such circumstances are discussed below.

If a tooth exhibits replacement resorption (ankylosis) it becomes impossible to move it orthodontically and infraocclusion of the tooth can be expected during the growth period of the alveolar process. In such cases, two major treatment options exist: either extraction combined with an alternative orthodontic treatment plan (eg combined with a restorative solution in conjunction with the patient's GDP) or maintaining the tooth, at least for some time, by repositioning it with the associated bone using a localised corticotomy when the patient's growth has stopped. ${ }^{8,9}$

Loss of vitality during orthodontic treatment means that treatment has to be temporarily interrupted whilst the patient is referred to their GDP for root canal treatment, and cannot be restarted until the endodontic therapy has been completed and the periapical lesion has healed.

Finally, it has been suggested ${ }^{10,11}$ that if signs of root resorption are observed radiographically after the first six to nine months of orthodontic treatment, radiographic controls should be undertaken over the following months, as the risk of severe root resorption appears high. However, a rest period of three months may help to reduce the degree of resorption..$^{8,12}$ Even so, severe root resorption must lead to a re-evaluation of the treatment goals and treatment plan.

To summarise, this patient was treated in a dental hospital and required only paedodontic advice, but it is evident that patients with traumatised teeth need to be treated effectively as joint cases. Input from the patient's GDP may be essential at various stages. For example, even when referring patients for orthodontic treatment, the details of any trauma the patient has sustained should be included where known, as the type of trauma may directly influence the risks involved in treatment. The GDP may also need to undertake the appropriate endodontic treatment should problems arise as the result of the original trauma during orthodontic treatment. They also have an important role to play in the long-term follow-up of those teeth affected by root resorption, as described above.

\section{Case 2}

A 13-year-old boy presented with a class II division 1 malocclusion associated with a class II skeletal pattern but with average vertical dimensions (Fig. 5). The lower lip was positioned just in front of the upper incisors.

Intra-orally, the upper incisors appeared normally inclined but the lower incisors were proclined. His overjet was $9 \mathrm{~mm}$ and the overbite was increased and complete to palate but atraumatic. All teeth were erupted except for the third molars. The upper arch was moderately crowded while the lower arch showed mild crowding. A marked enamel hypoplasia of all upper and lower incisors, canines and first premolars and upper second premolars was evident on examination. The dental history was positive for trauma to UL1 (21) which also had a blunt root but was vital.

The treatment plan involved extraction of upper first premolars and Tip-Edge fixed appliances in both arches. Following bonding the patient was instructed to wear class II intermaxillary elastics full-time. The patient proved a poor attendee but five months later, the overjet and overbite were satisfactorily reduced to edge-to-edge. Space closing mechanics were then commenced but unfortunately the upper labial segment became excessively retroclined. Regrettably, this remained unobserved for some three months as the patient missed a further appointment. He presented with an overjet of -1 to $-2 \mathrm{~mm}$, with one root tip being palpable in the upper labial sulcus. 


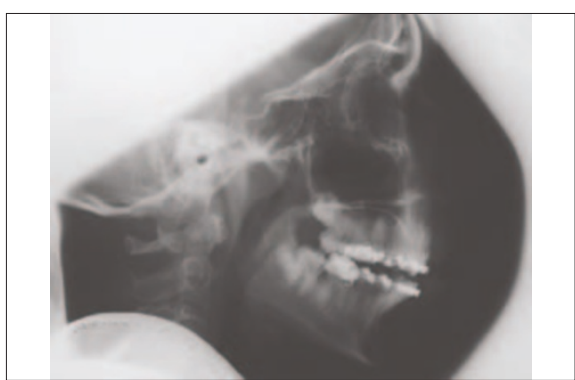

Fig 6 Case report 2: intra-treatment lateral cephalogram.

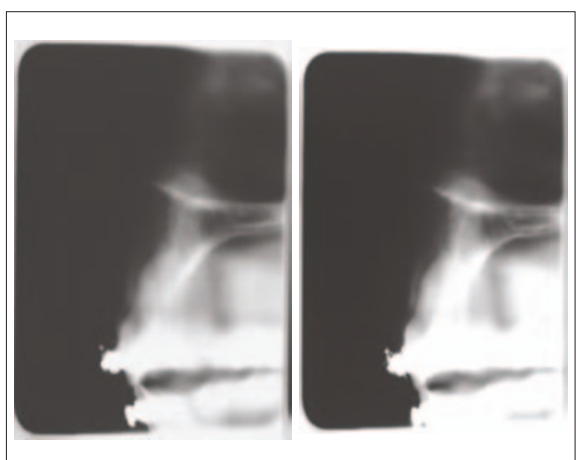

Fig 7a, 7b Case report 2: Scanora cross-sectional tomographs.

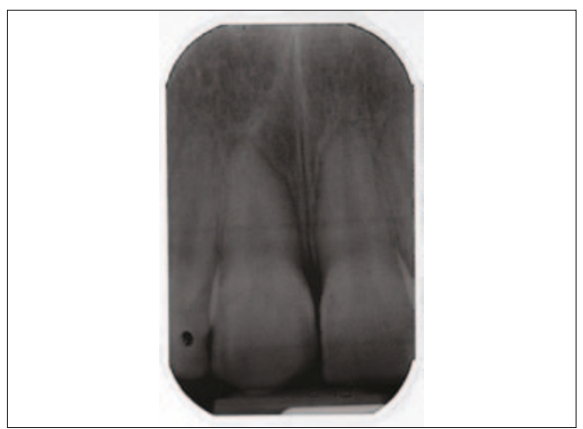

Fig 8 Case report 2: post-treatment periapical radiograph.

A lateral cephalometric radiograph confirmed that the root of UL1 (21) had perforated the buccal cortical plate (Fig. 6) and that the upper incisors were inclined at $71^{\circ}$ to the maxillary plane, yet both UL1 (21) and UR1 (11) were still vital. The need for improved cooperation and attendance was emphasised.

Four months later, a Scanora ${ }^{\circledR}$ crosssectional tomograph (Soredex, Onon Corporation, Helsinki, Finland) of both upper central incisors confirmed that the roots of both teeth lay once more within bone (Figs $7 \mathrm{a}, 7 \mathrm{~b})$. The overjet had also increased to $1 \mathrm{~mm}$. During this period, the patient's oral hygiene deteriorated and it was decided to simplify the mechanics by placing preadjusted brackets on the upper incisors and canines. The brackets were finally debonded 11 months later and upper and lower removable Hawley Retainers were fitted; the patient was advised to wear these six months full-time and six months nights only. At the end of retention, the occlusion was stable with bilateral class II molars and class I incisors. During the retention phase, radiographic checks of the upper incisors showed mild root resorption on the apical third of UL1 (21) (Fig. 8). The patient was discharged 18 months after debond back to his GDP.

Before orthodontic treatment is started, the borders of cortical bone should be estimated so that movements into the labial or lingual cortex are avoided, otherwise root resorption may result. $8,9,13$ The case presented is atypical as although one of the upper central incisors perforated the buccal cortical bone, no root resorption was observed nor loss of vitality at the end of treatment or even a year later. However, problems such as loss of vitality or/and root resorption could have arisen ${ }^{8,13}$ which might have delayed orthodontic treatment.

In summary, this patient missed eight orthodontic appointments including two hygienist appointments. His total active treatment time was two years, three months - a period clearly significantly lengthened by the poor attendance. Any patient undergoing orthodontic treatment should be registered with a GDP, but also go to their GDP regularly. The chance of improving treatment outcome could be enhanced by the referring GDP's help, ensuring that their patients are aware of the level of commitment that is required during orthodontic treatment, even prior to referral. It is also essential that all parts of the team see the patient regularly throughout their treatment in order to encourage and reinforce the need for cooperation in all aspects of their treatment. Furthermore, if the GDP is aware of factors that could impact negatively on the treatment outcome, then it is appropriate for the orthodontist to be informed.

\section{Case 3}

A 19-year-old girl presented a skeletally based class II division 2 malocclusion. She had a mild skeletal II pattern, associated with average vertical dimensions. Intra-orally her upper and lower incisors were retroclined. Her overjet was $7 \mathrm{~mm}$ and the overbite was increased but incomplete. There was a scissors bite at UR7 (17) and the buccal segments were bilaterally a half unit class II. Both arches were moderately crowded (Fig. 9). She had a history of loss of upper and lower first premolars, as part of a previous course of removable appliance orthodontic therapy. The incisor apex positions and root lengths were unclear on the cephalometric and on the upper anterior occlusal radiographs (Fig. 10).

Various treatment plans were discussed with the patient, but she opted for distal movement using headgear combined with

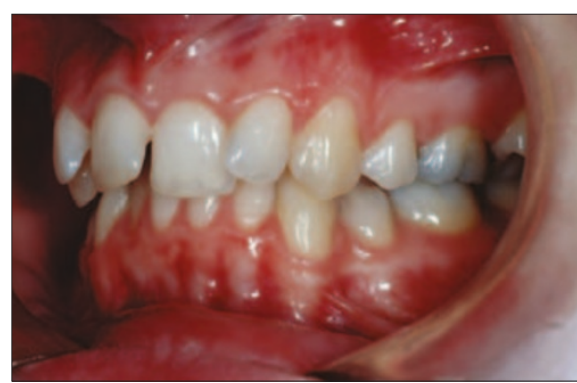

Fig 9 Case report 3: pre-treatment photograph.

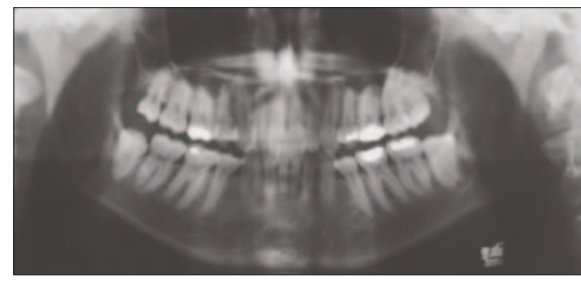

Fig 10 Case report 3: pre-treatment panoramic radiograph.

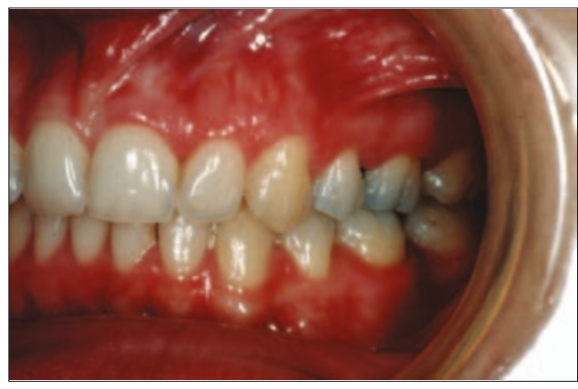

Fig 11 Case report 3: post-treatment photograph.

extractions and an upper removable 'Nudger' appliance carrying an anterior bite plane. The following teeth were extracted: UL7 (27), UR8 (18), LR7 (47) and LL8 (47), and ultimately, upper and lower pre-adjusted fixed appliances were placed.

After nine months of distal movement and overbite reduction, and following the extractions, the lower brackets were bonded. Seven months later when the molars were Class I and the overbite was fully controlled, the upper fixed appliance was placed. The upper fixed appliance phase took only 13 months and the total treatment time was two years, six months (Fig. 11).

At debond, upper and lower Hawley Retainers were fitted and the patient was advised to wear them full-time for six months and then nights only. After one year, the occlusion was stable with class I buccal and incisor relationships and referral back to the patient's GDP was considered. However, the patient mentioned incidentally that she had noticed some crossing over of the upper incisors when not wearing her upper Hawley Retainer and as indefinite retention was planned, a periapical radiograph was taken to check the upper incisor roots. A moderately severe root resorption of upper central and lateral incisors was found (Figs 12a, 12b). 


\section{PRACTICE}

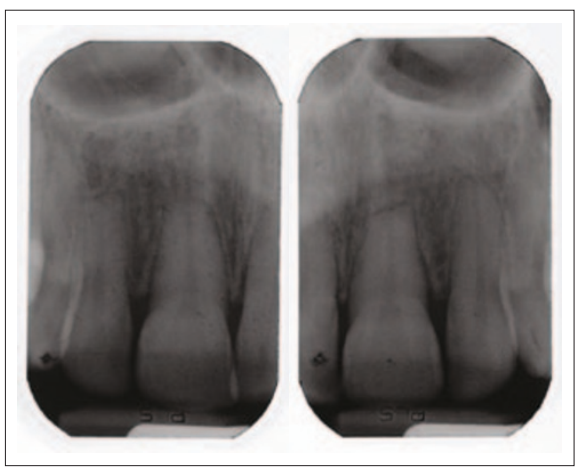

Fig 12a, 12b Case report 3: post-treatment periapical radiographs.

A new radiograph was taken three months later to assess whether the resorption was progressive in the light of the continuing tooth movement, as the possibility existed that further root resorption could occur due to 'jiggling' forces. No clear evidence of progression was seen. The patient declined use of bonded retainers and opted to wear the removable Hawley Retainers alternate nights indefinitely, although she was advised that if the root resorption did continue it would be necessary to stop the retainers and accept any relapse. Further follow-up showed a stable occlusion. Following discussion with the radiologist, it was decided to continue radiographic monitoring at increasing intervals. A new periapical was taken a year after the initial post-treatment one, and no changes were observed (Figs 13a, 13b).

In summary, long-term follow-up of root resorbed incisors indicates that after the appliances are removed, only remodelling of rough and sharp edges of the root occurs. ${ }^{14}$ Some tooth mobility may occur, but only when $9 \mathrm{~mm}$ or more of the root has resorbed. ${ }^{2}$

Long-term follow-up of these teeth by the GDP is recommended to ensure that root resorption is not progressing. The GDP should also consider endodontic treatment if it is required. ${ }^{2,14}$ Moreover, if severe root resorption occurs, then it will be particularly important that the patient maintains an excellent periodontal condition as otherwise the prognosis of the affected teeth is likely to be compromised.

This patient has not required treatment for the root resorption she experienced, but monitoring was required as the level of resorption could affect the longevity of her upper anterior teeth. This could be due to endodontic problems or perhaps more likely, if periodontal problems were to arise.

\section{DISCUSSION}

\section{The traumatised dentition}

Trauma to the upper incisors is common and has been particularly associated with increased overjet. ${ }^{15-17}$ It is therefore not surprising that many orthodontic patients will have traumatised upper incisors. It has recently been shown that while one upper incisor may sustain the brunt of a traumatic incident, 45\% of associated teeth will also have experienced some trauma at the same time. ${ }^{18}$ It is also therefore not surprising that the orthodontic management of such cases will frequently require a 'team effort' involving liaison between the orthodontist and the patient's GDP. Not only that, but the patient needs to be fully aware of the possible consequences not only of the orthodontic treatment itself but of the traumatic episode, generally since the repercussions may, in some circumstances, not appear until some years later, irrespective of any intervening orthodontic treatment. Should problems arise in treatment - requiring for example endodontic treatment - then treatment has to be stopped temporarily whilst the patient undergoes the required treatment (plus often a three month healing period) with their GDP. Treatment is thus prolonged, increasing the risk of other complications such as caries and periodontal problems. For these reasons it is essential that as far as is possible, dental health is as good as possible prior to beginning orthodontic treatment.

It has been recommended that patients with root fractures should have a follow-up period of at least two years before orthodontic treatment is commenced ${ }^{8,9}$ and inevitably, there will be limits imposed on the movement of these teeth. For example, they should be treated with light forces; use of removable appliances has been associated with reduced root resorption risk; ${ }^{1}$ prolonged tipping should be avoided and the treatment should be completed as quickly as possible. ${ }^{8,9,19}$ It is also necessary to monitor such teeth radiographically during treatment as well as monitoring their vitality. ${ }^{9}$ After orthodontic treatment has been completed, a period of retention similar to that of non-traumatised teeth has been recommended. ${ }^{8}$ In cases where one or more roots are fractured, a treatment involving functional appliances may therefore avoid or decrease the time required with fixed appliances.

\section{Risks associated with patient compliance}

Risks associated with patient compliance include patients whose oral hygiene deteriorates (precluding treatment progression) and those who fail to keep their follow-up appointments. Compliance is also required for the wearing of intra- or extra-oral auxiliaries needed to correct the malocclusion. The second patient clearly struggled in his ability to comply appropriately: failing appointments, failing to wear his elastics and later failing to control his oral hygiene.

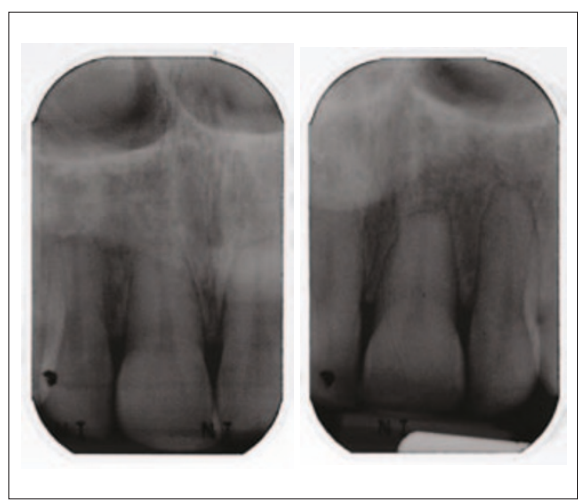

Fig 13a, 13b Case report 3: 1 year post-treatment periapical radiographs.

It is perhaps remarkable that in his case, the outcome was not more serious!

\section{Root resorption}

Numerous factors have been related to root resorption during orthodontic treatment. One factor is age at the start of treatment: patients starting treatment before 11 years of age have been found to have less root resorption than those who are treated later $^{1,20}$ and appears to be related to the possibility of continuing root development in the younger age group. An increased risk of root resorption with increasing length of active treatment has also been reported ${ }^{6}$ while some types of root shape - especially dilacerated and pipette-shaped roots have been associated with increased risk of root resorption. ${ }^{5,10}$ Some have also suggested that the amount of tooth movement may be a risk factor: Sameshima and Sinclair ${ }^{5}$ reported that the greater the overjet, the greater the root resorption for maxillary anterior teeth, since a greater root displacement requires a greater torque to correct the overjet. However, no such relationship was found by Linge and Linge ${ }^{1}$ whilse Kaley and Phillips ${ }^{21}$ found that class III patients suffered more root resorption possibly due to the proclination of the upper incisors to compensate for the class III skeletal pattern, forcing the roots against the lingual cortical plate. A similar situation could occur in patients with class II division 2 malocclusions. Previous trauma may also be a risk factor ${ }^{8}$ although not all studies confirm this. ${ }^{3,22}$ Nevertheless, traumatised teeth should always be evaluated carefully before proceeding with any orthodontic treatment, so that the risk of any complication is minimised. ${ }^{1,20,23}$

For the last case presented, it is probable that maxillary palatal root torque brought the upper incisors in to close proximity with the palatal cortex, and as has been described, root resorption of the maxillary incisors was induced ${ }^{1,13}$ as the cortical plates are another limit to tooth movement during orthodontic treatment. Despite this, without avoiding treatment altogether, it 
seems unlikely that where a patient appears to be susceptible, root resorption could have been avoided. It is evident that there is still much to be learnt about the causes of root resorption. The problem remains that increasing numbers of patients request or are advised to undertake permanent retention. Evidence as to the effects on the longterm progression of root resorption during retention with removable appliances is still required, but may become an increasing problem, especially if periodontal problems ensue as suggested by Vlaskalic and Boyd. ${ }^{7}$ In this case radiographic follow-up appears to be indicated although an established protocol is lacking.

\section{CONCLUSIONS}

For any orthodontic treatment to be carried out as successfully as possible, a team effort involving good three-way communication between the patient, the orthodontist and the general dental practice team is required. This is particularly true for patients presenting with some of the more unusual problems where orthodontic treatment may impinge on the treatment the GDP is required to offer and vice versa.

Thanks are due to Fiona Carmichael (Consultant Radiologist) for her helpful advice regarding radiographic follow up.
1 Linge B O, Linge L. Apical root resorption in upper anterior teeth. Eur J Orthod 1983; 5: 173-183.

2 Levander $E_{1}$ Malmgren 0. Long-term follow-up of maxillary incisors with severe apical root resorption. Eur J Orthod 2000; 22: 5-92.

3 Spurrier S W, Hall S H, Joondeph D R, Shapiro P A, Riedel R A. A comparison of apical root resorption during orthodontic treatment in endodontically treated and vital teeth. Am J Orthod Dentofacia Orthop 1990: 97: 130-134.

4 Mirabella A D, Artun J. Risk factors for apical root resorption of maxillary anterior teeth in adult orthodontic patients. Am J Orthod Dentofacial Orthop 1995; 108: 48-55.

5 Sameshima G T, Sinclair P M. Predicting and preventing root resorption: Part I. Diagnostic factors. Am J Orthod Dentofacial Orthop 2001; 119: 505-510.

6 Sameshima G T, Sinclair P M. Predicting and preventing root resorption: Part II. Treatment factors. Am J Orthod Dentofacial Orthop 2001: 119: 511-515.

7 Vlaskalic V, Boyd R L. Root resorptions and tissue changes during orthodontic Treatment. In Bishara $S$ E. (ed) Textbook of orthodontics. pp 463-475. Philadelphia: W B Saunders Company, 2001.

8 Malmgren O, Malmgren B, Goldson L. Orthodontic Management of the traumatized dentition. In Andreasen J 0, Andreasen F M. (eds) Textbook and color atlas of traumatic injuries to the teeth, $3^{\text {rd }} \mathrm{ed}$. pp 587-633. Copenhagen: Mosby, 1994.

9 Attack K. The orthodontic implications of traumatized upper incisor teeth. Dent Update 1999; 26: 432-437.

10 Levander E, Malmgren O. Evaluation of the risk of root resorption during orthodontic treatment: A study of upper incisors. Eur J Orthod 1988; 10: 30-38.

11 Orthodontic Radiography Guidelines. BOS (ed) 2001.

12 Levander E, Malmgren O, Eliasson S. Evaluation of root resorption in relation to two orthodontic treatment regimes. A clinical experimental study. Eur J Orthod 1994; 16: 223-228.
13 Proffit W R, Fields H W. Contemporary orthodontics ( $3^{\text {rd }}$ ed). St Louis, Missouri: Mosby, 1999.

14 Remington D N, Joondeph D R, Artun J, Riedel R A Chapko M K. Long-term evaluation of root resorption occurring during orthodontic treatment. Am J Orthod Dentofacial Orthop 1989: 96: 43-46.

15 Burden D J. An investigation of the association between overjet size, lip coverage and traumatic injury to maxillary incisors. Eur J Orthod 1995; 17: 513-517.

16 Nguyen QV, Bezemer P D, Habets L, Prahl-Andersen B. A systematic review of the relationship between overjet size and traumatic dental injuries. Eur J Orthod 1999; 21: 503-515.

17 Cortes M I, Marcenes W, Sheiham A. Prevalence and correlates of traumatic injuries to the permanent teeth of schoolchildren aged 9-14 years in Belo Horizonte, Brazil. Dent Traumatol 2001; 17: 22-26.

18 Majorana A, Pasini S, Bardellini E, Keller E. Clinical and epidemiological study of traumatic root fractures. Dent Traumato/ 2002; 18: 77-80.

19 Drysdale C, Gibbs S L, Pitt Ford T R. Orthodontic management of root-filled teeth. Br J Orthod 1996; 23: 255-260

20 Mavragani M, Vergari A, Selliseth $N$, Boe O E Wisth $\mathrm{P}$ J. A radiographic comparison of apical root resorption after orthodontic treatment with a standard edgewise and straight-wire edgewise technique. Eur J Orthod 2000; 22: 665-674.

21 Kaley J, Phillips C. Factors related to root resorption in edgewise practice. Angle Orthod 1991; 61: 125-132.

22 Malmgren 0 , Goldson L, Hill C, Orwin A, Petrini L Lundberg M. Root resorption after orthodontic treatment of traumatized teeth. Am J Orthod Dentofacial Orthop 1982; 82: 487-491.

23 Linge L. Patient characteristics and treatment variables associated with apical root resorption during orthodontic treatment. Am J Orthod Dentofacial Orthop 1991; 99: 35-43. 